\title{
La enseñanza de la historia y las ciencias sociales en la educación primaria en Colombia: de las políticas curriculares a las prácticas educativas
}

\section{O ensino de história e ciências sociais na educação primária na Colômbia: das políticas curriculares às práticas educacionais}

\section{The teaching of history and social sciences in primary education in Colombia: from curricular policies to educational practices}

\author{
Diana Marcela Arana Hernández* \\ Martha Cecilia Gutiérrez Giraldo*
}

\begin{abstract}
RESUMEN
El artículo presenta una síntesis de lo que ha sido la enseñanza de la historia y de las ciencias sociales en Colombia desde las políticas gubernamentales de las últimas décadas. A partir de los propósitos enunciados en estas políticas, se analiza lo que pasa en dos casos conformados por profesoras noveles de básica primaria que enseñan en instituciones educativas públicas; se relaciona lo que ellas piensan con lo que hacen en las prácticas, respecto a las finalidades de la enseñanza de las ciencias sociales. Los resultados muestran dos experiencias que coinciden en las finalidades que las profesoras se proponen (de corte crítico), y difieren en las finalidades que se evidencian en sus prácticas (una positivista y la otra crítica). Los hallazgos generan cuestionamientos frente al sentido de los debates de las políticas
\end{abstract}

*Universidad Tecnológica de Pereira. Pereira, Risaralda, Colombia. Email: dmarana@utp. edu.co - https://orcid.org/0000-0003-4359-1720 Email: mgutierrez@utp.edu.co - https://orcid. org/0000-0002-1221-2137 
gubernamentales para la enseñanza de las ciencias sociales, y lo que ocurre durante la formación inicial de docentes generalistas para la enseñanza de estas ciencias. El debate debe enfocarse en los propósitos de la enseñanza de la historia y de las ciencias sociales, para que propicie prácticas educativas innovadoras y coherentes.

Palabras clave: Finalidades de la enseñanza de la historia. Prácticas educativas. Educación básica primaria. Docentes noveles. Formación inicial docente.

\title{
RESUMO
}

$\mathrm{O}$ artigo apresenta uma síntese do que tem sido o ensino de história e ciências sociais na Colômbia tendo em vista as políticas governamentais das últimas décadas. A partir dos propósitos previstos nessas políticas, analisa-se o que ocorre no estudo de caso de dois professores iniciantes do ensino fundamental que lecionam em instituições públicas de ensino: o que pensam está relacionado com o que fazem na prática, no que diz respeito aos fins de ensino das ciências sociais. Os resultados mostram duas experiências que coincidem nos propósitos que os professores propõem (críticos) e se diferenciam nos propósitos que se evidenciam em suas práticas (um positivista e outro crítico). Os resultados levantam questionamentos sobre o significado dos debates sobre as políticas governamentais para o ensino das ciências sociais e o que ocorre com a formação inicial de professores generalistas para o ensino dessas ciências. A discussão deve centrar-se nos propósitos do ensino de História e das Ciências Sociais, de modo a fomentar práticas educativas inovadoras e coerentes.

Palavras-chave: Finalidades do ensino de história. Práticas educativas. Educação básica. Professores iniciantes. Formação inicial de professores.

\begin{abstract}
The article presents a synthesis of what has been the teaching of history and social sciences in Colombia from the government policies of the last decades. Based on the purposes stated in these policies, it is analyzed what occurs in two cases conformed by novice elementary school teachers who teach in public schools; what they think is related to what they do in practice, with regard to the purposes of teaching social sciences. The results show two experiences that coincide in the purposes that the teachers propose (critical) and differ in the purposes that are evidenced in their practices (one positivist and the other critical). The results raise questions about the meaning of the
\end{abstract}


debates of government policies for the teaching of social sciences and what happens with the initial training of generalist teachers for science teaching. The debate should focus on the purposes of the teaching of history and social sciences, so that it fosters innovative and coherent educational practices.

Keywords: Purposes of the teaching of history. Educational practices. Primary education. Novice teachers. Initial teacher training.

\section{Introducción}

La enseñanza de la historia y de las ciencias sociales en Colombia ha sido objeto de debates enfocados en la organización de las disciplinas y de los contenidos escolares. Estas polémicas han aportado poco a la transformación de las prácticas educativas.

\section{La enseñanza de la historia y las ciencias sociales en Colombia}

En Colombia, los propósitos que han orientado la enseñanza de las ciencias sociales y de la historia, son el reflejo de las tensiones registradas a nivel social y político desde tiempo atrás. Estas tensiones se evidencian en los documentos oficiales que se describen a continuación.

Desde el siglo XIX, la enseñanza de la historia estuvo al servicio del Estados-nación y, por tanto, fue forjadora de identidad y valores patrios (SÁNCHEZ VÁSQUEZ, 2013). La escuela

[...] se limitaba a reproducir en el aula de clase el conocimiento producido por los historiadores, el cual se contaba como si fuera una verdad incuestionable y que, como tal, exigía del estudiante no tanto su comprensión sino su memorización (RODRÍGUEZ; GARZÓN, 2004, p. 24). 
Esta forma de entender la enseñanza corresponde a una concepción positivista de la sociedad, desde la cual los hechos hablan por sí mismos y se encuentran objetivamente en los documentos. En esta misma dirección, Durkheim (1986) afirma que los hechos sociales deben ser considerados como cosas, como realidades externas, tanto de los individuos a los que afecta, como de quienes los estudian.

A principios del siglo XX, el gobierno colombiano vio en la enseñanza de la historia el medio estratégico para impulsar la unificación nacional. En esta época se fundó la Academia Colombiana de Historia ( $\mathrm{ACH})$, encargada de divulgar la memoria oficial del país. El Gobierno expidió el decreto 2388 de 1948, que intensificaba la enseñanza de la historia patria, considerando que el conocimiento de esta disciplina, el culto a los próceres y la veneración por los símbolos de la nacionalidad eran elementos inapreciables de cohesión nacional y de dignidad ciudadana (REPÚBLICA DE COLOMBIA, 1948). Así se instauró el estudio de la historia de Colombia de manera obligatoria (mínimo dos años en la educación básica primaria y dos en secundaria). A la ACH le correspondía la vigilancia de los programas, los textos y los elementos vinculados a la enseñanza de la historia en los establecimientos educativos del país.

Tras dicha reglamentación para la enseñanza de la historia, cuya finalidad era promover la adquisición por parte de los estudiantes de ciertos valores nacionalistas a través de la enseñanza y el aprendizaje de piezas de información objetivas, se encuentra la creencia de que cierta información fáctica es importante para la práctica de la buena ciudadanía lo que corresponde a una de las tradiciones en el campo del currículo de los estudios sociales citadas por Ross (2020, p. 6), denominada "social studies as citizenship (or cultural) transmission" [Estudios sociales como transmisión de ciudadanía (o cultural)].

Hacia la década de 1970, con la influencia de historiadores colombianos, quienes bajo el influjo de la profesionalización de las ciencias sociales llegaron al país después de formarse en Europa y en Estados Unidos en tradiciones como la escuela de los annales francesa o la escuela tradicional marxista inglesa, surgió una nueva generación que empezó a escribir la Nueva Historia de Colombia (SÁNCHEZ VÁSQUEZ, 2013).

Los académicos de la Nueva Historia fueron invitados a participar en la construcción de los marcos generales de los programas curriculares para la enseñanza de las ciencias sociales, lo que generó confrontaciones entre los intelectuales de la disciplina histórica que, de uno y otro lado, defendían una forma específica de concebir y producir la historia (ARIAS, 2015).

En la década de 1980, el Ministerio de Educación Nacional (MEN), con la asesoría de la Unesco, propuso la integración al interior de las áreas de conocimiento y entre diferentes asignaturas para disolver las barreras 
disciplinares existentes. Este enfoque integrador se justificó con razones psicológicas y sociales para optar por una mirada globalizadora de la educación. En el año de 1984, el gobierno expidió el decreto 1002 (REPÚBLICA DE COLOMBIA, 1984) a través del cual se crearon oficialmente las ciencias integradas, tanto naturales como sociales. En el caso de las ciencias sociales "el objetivo central fue acompasar los últimos logros en el saber especializado con la comprensión de la realidad nacional por parte de los educandos" (ACEVEDO; SAMACÁ, 2012, p. 237). Estas transformaciones se realizaron en consonancia con un enfoque interpretativo y crítico de la investigación social, en reacción a la concepción positivista de la ciencia.

La integración de las ciencias suscitó el rechazo de las miradas fragmentadas del conocimiento social y la enseñanza de las disciplinas de manera aislada. Así, la historia y la geografía pasaron a integrarse a una nueva área llamada Ciencias sociales. En 1991, con la promulgación de la Constitución Política de Colombia actual (REPÚBLICA DE COLOMBIA, 1991), se emprendió una reforma integral del sistema educativo que dio lugar a la Ley General de Educación (Ley 115 de 1994) (REPÚBLICA DE COLOMBIA, 1994), en la que se especifica como finalidad central de la enseñanza obligatoria la formación de ciudadanos; se establece el área de las Ciencias sociales como área obligatoria y fundamental, integrada por: Historia, Geografía, Constitución política y democracia (Ley 115 de 1994, art. 23) (REPÚBLICA DE COLOMBIA, 1994).

Pese a que esta ley apostó por unas ciencias sociales integradas, en la práctica se continuó dando mayor peso a la historia y en menor medida a la geografía, sin que se evidenciara una noción integral de la enseñanza de las Ciencias sociales. Según el MEN (2002), esto se debió a que casi todos los docentes del país habían sido formados en la universidad con un enfoque disciplinar y también, porque "muchos de ellos llevaban años trabajando desde el enfoque histórico y geográfico, que contribuía a reforzar los libros de texto producidos hasta ese momento" (REPÚBLICA DE COLOMBIA, 2002, p. 11).

En el año 2002, el MEN publicó los Lineamientos curriculares del área de Ciencias sociales para la Educación básica y media del país (REPÚBLICA DE COLOMBIA, 2002), caracterizados por la flexibilidad e integración del conocimiento social a través de ejes generadores que buscaban promover la formación de ciudadanos críticos, democráticos y solidarios frente a los problemas de la sociedad local, nacional y global, y a sus posibles soluciones. Entre los fundamentos teóricos que orientan estos lineamientos curriculares están Gibbons et al. (1997) y Dogan y Pahre (1993) quienes coinciden en plantear la intersección entre las disciplinas como innovación en las ciencias sociales, que complementa el previo proceso de fragmentación y especialización que ha caracterizado la segundo mitad del siglo XX. La propuesta integradora 
de los lineamientos, en consonancia con unas ciencias sociales abiertas (WALLERSTEIN, 1999), pretendía que los estudiantes comprendieran la realidad social transcendiendo las fronteras de las disciplinas.

Posteriormente, con la publicación de los Estándares Básicos de Competencias por parte del MEN (REPÚBLICA DE COLOMBIA, 2006), se buscó establecer referentes comunes sobre lo que deben aprender los estudiantes por conjuntos de grados en la educación básica y media, lo que puso en entredicho la coherencia con las políticas educativas anteriores, puesto que la Ley 115 de 1994 (REPÚBLICA DE COLOMBIA, 1994) da autonomía a las instituciones para la construcción de sus currículos a través de proyectos educativos institucionales. El propósito de dichos estándares fue establecer unos referentes comunes para mejorar la calidad de la educación y responder a requerimientos de evaluaciones nacionales e internacionales, que acaban con la autonomía en la toma de decisiones curriculares por parte de las instituciones educativas y de los profesores.

Según Orozco (2004), las reformas relacionadas con las competencias en la evaluación de los aprendizajes escolares están alimentadas por la ideología neoconservadora que apuntala el proyecto económico neoliberal, y que pretende asegurar el control político del saber para homogeneizar la educación según esquemas extranjeros. Explica que estas reformas fomentan el reduccionismo de unos currículos vistos como obligatorios a escala nacional, restringen la autonomía y la innovación docente, frente a las ambiciones económicas de los mercados editoriales, entre otros.

Posteriormente, en el año 2016, el MEN publicó los Derechos Básicos de Aprendizaje (DBA) (REPÚBLICA DE COLOMBIA, 2016) que definen los aprendizajes estructurantes que han de aprender los estudiantes en cada grado escolar, desde transición a grado once, en el área de Ciencias sociales. Esto acentuó el problema de la autonomía docente defendida en los lineamientos curriculares formulados en el 2002.

La reglamentación anterior fomentó la discusión en la comunidad académica nacional, especialmente de los historiadores del país, para quienes existe un desconocimiento de la historia por parte de las nuevas generaciones. Tal situación llevó a la promulgación de la Ley 1874 de 2017 (REPÚBLICA DE COLOMBIA, 2017), con el objeto de restablecer la enseñanza obligatoria de la Historia de Colombia como una disciplina integrada en los lineamientos curriculares de las ciencias sociales en la educación básica y media.

Sin embargo, el problema no está en el "restablecimiento" de la historia como lo dice la ley citada, porque conocer la historia de manera exhaustiva en la escuela no garantiza la formación de ciudadanos democráticos. La reflexión debe girar en torno a por qué y para qué enseñar historia, cómo vincular esos 
propósitos al currículo de las ciencias sociales con el fin de que el estudiante pueda utilizar esa historia para problematizar el presente, comprender el pasado y pensar en las posibilidades que tiene para construir el futuro. El asunto no es de más o menos historia, sino de responder al por qué y para qué del conocimiento que se desea introducir en la escuela, llámese historia, ciencias sociales o estudios sociales (ARIAS, 2015).

Pensar en los propósitos de la enseñanza de la historia y de las ciencias sociales en la escuela, lleva a cuestionar qué está pasando con la formación de profesores de ciencias sociales y, específicamente, con los de educación básica primaria que son generalistas; es decir, que se preparan para enseñar todas las áreas del conocimiento en esta etapa educativa y deben ser capaces de tomar decisiones sobre el conjunto de áreas y disciplinas a enseñar.

Ante el panorama descrito, cabe preguntarse si las universidades forman maestros de básica primaria para la toma de decisiones en la selección y organización de los contenidos escolares, con unas finalidades que respondan a la integración de las ciencias sociales propuesta en la Ley 115 de 1994 (REPÚBLICA DE COLOMBIA, 1994) y en los lineamientos curriculares de 2002 (REPÚBLICA DE COLOMBIA, 2002), que propenden por la formación de ciudadanos críticos, capaces de comprender la realidad y aportar a su trasformación.

Los programas universitarios de formación inicial del profesorado incluyen un componente disciplinar fuerte en la preparación de licenciados en Ciencias sociales, Historia, Geografía y Ciencias Económicas y Políticas. Sin embargo, en los programas de licenciatura para la educación preescolar y básica primaria (Licenciatura en Educación Infantil o Licenciatura en Educación Básica Primaria), el énfasis se da en el componente pedagógico y didáctico, lo que hace más compleja la toma de decisiones curriculares de los profesores generalistas, ante la falta de profundidad en el conocimiento disciplinar.

Además de la dificultad mencionada, de acuerdo con Levstik y Tyson (2008), el profesor generalista en los primeros años de actividad profesional, se enfrenta a situaciones de incertidumbre por la desconexión entre la formación recibida en la universidad y la realidad de lo que sucede en el aula (LEVSTIK; TYSON, 2008). Según Porlán (2017), los profesores noveles consideran que los conocimientos sobre la enseñanza y el aprendizaje aprendidos en la universidad son poco efectivos en la práctica.

La problemática sobre la desconexión entre la formación universitaria y la práctica profesional de los profesores noveles generalistas que enseñan ciencias sociales en la educación básica primaria, lleva a profundizar en lo que pasa cuando ellos ejercen su profesión, después de haber culminado la formación inicial en la que, se supone, debieron construir el conocimiento base necesario para la enseñanza (SHULMAN, 2005). Además, es preciso considerar la 
pregunta por las finalidades de la enseñanza de las ciencias sociales enunciadas en los lineamientos curriculares del área, que buscan ayudar a los estudiantes a comprender la realidad nacional (pasado-presente) para transformar la sociedad con una consciencia crítica, solidaria y respetuosa de la diferencia y la diversidad.

Con el propósito de contribuir a la reflexión sobre las finalidades expresadas en las políticas educativas colombianas y su relación con lo que pasa en las prácticas educativas de los profesores de primaria, se propone la investigación en la que se enmarca este artículo, cuyo objetivo es interpretar las relaciones entre lo que los profesores noveles generalistas piensan acerca de las finalidades de la enseñanza de las ciencias sociales, y lo que hacen en sus prácticas educativas.

Para el análisis se tiene en cuenta las finalidades que subyacen a las políticas educativas y a las prácticas de las profesoras noveles. Específicamente, se hace referencia a las finalidades de la educación social (BENEJAM, 2002), a las tradiciones curriculares para la enseñanza de los estudios sociales (ROSS, 2020) y a los enfoques de la enseñanza de los social studies [estudios sociales] identificados por Goodman y Adler (1985).

\section{Metodología}

La investigación se ubica en el enfoque hermenéutico, busca analizar e interpretar las relaciones entre lo que los profesores noveles piensan sobre las finalidades de la enseñanza de las ciencias sociales, y lo que hacen en su ejercicio profesional cuando enseñan estas ciencias en la educación básica primaria.

La estrategia es un estudio de casos (YIN, 2009), en el que se describe, analiza e interpreta una realidad compleja como lo es la enseñanza de las ciencias sociales en su contexto natural. Los casos fueron seleccionados mediante un muestreo intencional (PATTON, 1990). En este artículo se presentan los resultados de dos de los cuatro casos de la investigación: el caso de la profesora con mayor experiencia y el de la profesora con menor experiencia. La unidad de análisis corresponde a la identificación, análisis e interpretación de las finalidades de la enseñanza de las ciencias sociales expresadas por las profesoras, y las desplegadas en sus prácticas de enseñanza en la educación básica primaria.

El caso 1 corresponde a una profesora de 27 años de edad, egresada de un programa de licenciatura en pedagogía infantil de una universidad pública colombiana. Al inicio de la investigación tenía cuatro años de experiencia y trabajaba en una institución educativa pública de un municipio de la zona cafetera colombiana. 
El caso 2 corresponde a una profesora de 22 años de edad, egresada del mismo programa y universidad. Al inicio de la investigación se encontraba en su primer año de ejercicio profesional, trabajando en una sede rural de una institución educativa pública ubicada en un municipio de la zona cafetera colombiana.

El procedimiento para la recolección de la información consta de dos momentos: antes de la práctica, se realiza una entrevista semiestructurada (FLICK, 2012) a cada profesora para conocer lo que piensa sobre las finalidades de la enseñanza de las ciencias sociales y la forma de enseñarlas. Durante la práctica educativa, se hace observación no participante al desarrollo de las unidades didácticas. Las clases se registran en audio y video; la planeación docente y la producción de los estudiantes hacen parte del material complementario.

La información obtenida en las entrevistas y en la observación, se analiza mediante la técnica de codificación teórica (FLICK, 2012; STRAUSS; CORBIN, 2012), que incluye la codificación abierta (fragmentación y conceptualización de los datos), la codificación axial (identificación de categorías) y la codificación selectiva (interpretación de las categorías). Para interpretar las relaciones entre los resultados obtenidos en los dos momentos, se utiliza la técnica de triangulación mixta (FLICK, 2014), que permite analizar las relaciones entre las finalidades de las ciencias sociales que expresan y evidencian las profesoras en sus prácticas.

\section{Resultados}

De acuerdo con el procedimiento descrito, en el primer momento del análisis se identificó lo que cada una de las profesoras piensa y dice sobre las finalidades de la enseñanza de las ciencias sociales y la forma de enseñarlas, con los resultados que se exponen a continuación.

La profesora del caso 1 afirma que la enseñanza de las ciencias sociales debe partir de la observación del contexto. Esto lo ejemplifica así:

Para el niño es común ver la guerrilla, ver la contaminación de su río, pero para mi no es común porque no es mi contexto, pero estoy dentro de ese contexto, tengo que identificar los puntos problemáticos y que ellos están acostumbrados, para trabajar desde allí. 
Para ella, la enseñanza de las ciencias sociales debe hacerse desde situaciones problema reales, con el fin de que los estudiantes se ubiquen en su contexto, desarrollen competencias ciudadanas que les permita resolver sus conflictos de manera pacífica, a través del diálogo, respetándose a sí mismos y a los demás.

La profesora del caso 2 piensa que es fundamental abordar en el aula

los efectos de las actividades económicas que ellos manejan [los estudiantes y familias del sector rural en que se encuentran]; cómo eso influye en su contexto, cómo pueden generarse nuevas prácticas económicas... sustentables en el caso de la región [...]. Otros contenidos que deberían plantearse es la manera en que ellos pueden ayudar a conservar todo el patrimonio que tienen, específicamente La Celia y la región cafetera, de la parte tanto cultural, la historia, los paisajes; que sepan qué es lo que todo eso significa y más ahora que los pueblos se están volviendo tan turísticos.

Para la profesora de este caso, lo importante es buscar que los estudiantes sean conscientes de cuál es su entorno y puedan intervenir en él:

Porque muchos de los niños, por la misma manera en que se ha trabajado, no comprenden bien la relación entre lo que les enseña la escuela y lo que sucede en los contextos, eso no es solo en ciencias sociales... por ejemplo, si vieron cuáles son los gases de efecto invernadero, los efectos de su uso, que sean capaces de decir: papá vea, nosotros vimos en la escuela esto $y$ vimos que este tipo de fertilizantes pues no se debe utilizar por esto...

La professora también expresa: "Es necesario reorientar la enseñanza de las ciencias sociales hacia la formación de ciudadanos comprometidos con la comprensión del medio social y su transformación".

En síntesis, en lo que las profesoras manifiestan se identifican propósitos en la enseñanza de las ciencias sociales relacionados con el reconocimiento de los contextos y la solución pacífica de conflictos (caso 1); y, el desarrollo de habilidades que ayuden a los estudiantes a comprender el mundo en que viven y actuar en él para transformarlo (caso 2). De acuerdo con sus declaraciones, las finalidades que guían la enseñanza del conocimiento social de estas profesoras noveles, se aproximan a un enfoque crítico; reconocen la relevancia de trabajar 
a partir de problemas sociales cercanos al contexto de los estudiantes y hablan de la importancia de que ellos lleguen a actuar en sus entornos para provocar cambios en favor de sus comunidades.

En el segundo momento del análisis, relacionado con las finalidades vehiculadas en las prácticas de enseñanza de las ciencias sociales, se encuentra lo siguiente:

En el caso 1, la unidad didáctica se desarrolla en una institución educativa urbana con estudiantes de grado cuarto de básica primaria. El tema central es: "Las regiones naturales de Colombia". La profesora se enfoca en la enseñanza de contenidos declarativos en el marco de un proyecto cuyo producto final es la elaboración de materiales informativos sobre el tema, para ser expuestos por los estudiantes ante la comunidad educativa. Su objetivo es que los estudiantes identifiquen las características de las regiones naturales de Colombia a partir de la información presentada en diferentes medios (videos, textos, mapas), la escriban en sus cuadernos y la socialicen. De acuerdo con lo observado e identificado en esta práctica, el fin de la enseñanza de las ciencias sociales es reproducir el conocimiento.

En las clases se observa la transmisión y control total del proceso por parte de la profesora, quien plantea el trabajo en grupo para que los estudiantes respondan a lo propuesto respecto al manejo de información y materiales. Controla la participación y los estudiantes interactúan en el manejo de la información para realizar los productos solicitados. Quienes no cumplen las normas son sancionados o amenazados con sanciones, lo cual genera una relación tensa entre estudiantes y profesora, quien hace continuos llamados de atención ante la desobediencia o por comportamientos que le incomodan.

En este caso se evidencia una práctica tradicional, que desconoce el contexto de los estudiantes. No hay construcción de conocimientos; la profesora espera que ellos los adquieran a partir del uso que hacen de la información proporcionada (lectura, reescritura -transcripción - y socialización). Existe una relación vertical entre la profesora y los estudiantes, centrada en la disciplina y el control. El resultado del proceso de análisis de esta práctica permite identificar una "enseñanza directiva de contenidos declarativos sobre las regiones naturales de Colombia", que corresponde a un enfoque positivista de la enseñanza de las ciencias sociales.

En el caso 2, la unidad didáctica se desarrolla en un aula multigrado de una institución educativa rural, donde convergen estudiantes de diferentes grados (de transición a grado quinto de básica primaria). El tema central es el calentamiento global. El objetivo de la profesora, según se observó, es que los estudiantes analicen las causas y consecuencias de un problema socioambiental (calentamiento global), hasta llegar a comprometerse con posibles acciones para 
solucionarlo. Esto denota interés por el desarrollo de habilidades de pensamiento a partir del problema socioambiental (privilegio de finalidades intelectuales). Para lograrlo, la profesora promueve conversaciones en gran grupo a partir de imágenes que representan situaciones reales relacionadas con los contenidos de clase (causas y consecuencias del problema).

Durante las clases se presentan unos momentos secuenciales que definen el curso de acción de la profesora. Generalmente inicia con una introducción para establecer conexión con los temas ya estudiados. A veces la socialización de las tareas es útil para establecer relaciones con conceptos nuevos. Hay un momento central en la clase que corresponde a las actividades individuales o en pequeños grupos, o dirigidas al gran grupo. A partir de imágenes, videos, textos o de una salida pedagógica, la profesora propone actividades diferenciadas según el nivel escolar de los estudiantes.

La conformación de los pequeños grupos varía según el nivel escolar. La mayoría de las veces se conforman tres grupos: los estudiantes de transición y primero; los de segundo y tercero, y los de cuarto y quinto grado. Otras veces se conforman grupos heterogéneos buscando apoyo y ayuda mutua entre compañeros de distintas edades y nivel escolar.

Al final de las clases, la profesora suele asignar tareas para la casa que, al igual que las actividades en la escuela, tienen como propósito aplicar lo aprendido (identificar y representar acciones en las que se producen determinados gases de efecto invernadero), profundizar en los contenidos vistos (leer información sobre el uso y aprovechamiento de los desechos del café y reflexionar sobre ello) y, además, vincular a las familias.

En las actividades propuestas en la clase, la profesora ofrece apoyo y ayuda constante y simultánea a los pequeños grupos, y resuelve inquietudes de los estudiantes. Estos, a su vez, responden a las preguntas de la profesora, resuelven las actividades, expresan sus dificultades, relacionan la información de clase con sus contextos (aportan información desde sus experiencias e identifican causas y consecuencias del problema socioambiental en sus contextos), ayudan a otros compañeros, socializan sus tareas y eligen posibles soluciones al problema que pueden aplicar en sus contextos.

El análisis de las causas del problema abordado permite a los estudiantes reconocerse como parte del problema, especialmente con el uso que hacen en sus fincas de los desechos del café, de herbicidas, fertilizantes y con la ganadería extensiva. Al abordar las consecuencias del problema para la naturaleza y para los seres humanos, desde la observación de imágenes que representan la realidad, la profesora logró el objetivo de sensibilizar a los estudiantes frente al mismo, convirtiéndose en la base para que ellos propusieran posibles soluciones. En la observación se identifican otros logros de la profesora: integró las ciencias 
naturales con las ciencias sociales en torno al problema abordado; la evaluación fue parte del proceso de formación; tuvo en cuenta las capacidades y la singularidad de los estudiantes, y revisó continuamente el avance individual de ellos (en las diferentes actividades propuestas).

En definitiva, se observa una práctica guiada en la que la profesora interactúa con los estudiantes en la realización de actividades que apuntan al cumplimiento de los propósitos de la clase. Atiende las solicitudes de los estudiantes estableciendo una relación de respeto y trato amable. También se observa un trabajo cooperativo entre los estudiantes del mismo nivel escolar y entre algunos de nivel superior con los más pequeños.

El análisis realizado en este segundo momento a los dos casos, permite vislumbrar las tradiciones y tendencias de mayor influencia en estas prácticas. El caso 1 está influenciado por la tradición positivista de la enseñanza de las ciencias sociales, porque sigue un programa exhaustivo de objetivos y de contenidos secuenciados con la intención de que los estudiantes conserven el orden planeado. De acuerdo con la tradición positivista, el mundo es objetivo y mesurable, de manera que la persona puede llegar a comprenderlo mediante la observación y el razonamiento (BENEJAM, 2002). La profesora pretende que los estudiantes memoricen el conocimiento que es objeto de enseñanza y aprendizaje, para lo cual propone diferentes estrategias que terminan en la reproducción de los contenidos tal como se presentaron.

Las actuaciones de esta profesora, influenciadas por el modelo tecnicista (BENEJAM, 2002), dejan ver una práctica educativa tradicional. Ella es quien proporciona un saber válido, para lo cual emplea diferentes medios (fotocopias de libros de texto, videos, esquemas), con el fin de que los estudiantes lo repitan y representen en otros medios (carteleras, cartillas, juegos de roles). La enseñanza de las ciencias sociales en este modelo, enseñar para adoctrinar, tiene como finalidad "formar ciudadanos con la conciencia de pertenecer a un país o nación” (SANTISTEBAN; PAGÉS, 2011, p. 51), idea que se explicita en el objetivo que tiene la profesora para la unidad: "Reconocer las características de cada una de las regiones de nuestro país, con el fin de crear un sentido de identidad y pertenencia por todo lo que nos rodea" (caso 1, clase 1). En esta práctica, se entiende la ciudadanía como la adquisición de valores y contenidos preestablecidos mediante un consenso de autoridades y expertos, lo que corresponde a una perspectiva curricular de los estudios sociales como transmisión de ciudadanía (o cultural), orientadora de políticas anteriores y dominante durante mucho tiempo, que ha experimentado un resurgimiento (ROSS, 2020). 
Por su parte, la práctica de la profesora del caso 2 se aproxima a un enfoque crítico. Incluye como contenido central un problema socioambiental (calentamiento global) que puede considerarse como un problema social relevante, en torno al cual integra la enseñanza de diferentes disciplinas buscando que los estudiantes se comprometan con acciones que permitan afrontarlo y aporten a su solución. La concepción de ciudadanía que subyace en este caso, se ubica en los estudios sociales como indagación reflexiva, enfatiza en la resolución de problemas relevantes o la toma de decisiones significativas dentro de un contexto específico, (ROSS, 2020).

De acuerdo con Santisteban y Pagés (2011, p. 53), las

Ciencias sociales deben aportar al alumnado los conocimientos, las actitudes y las técnicas para la acción, que le permitan situarse ante los problemas sociales del pasado y del presente, evaluarlos e intervenir en su gestión, para ir construyendo el futuro.

Este propósito es el que se devela en la práctica de la profesora del caso 2, pues lleva a los estudiantes a comprender el problema del calentamiento global a partir de la observación de imágenes. Promueve la reflexión sobre qué pudo ocasionar esas situaciones (muerte de animales, desastres naturales, contaminación, entre otros), para luego profundizar en el conocimiento de las causas, específicamente desde el conocimiento de los gases de efecto invernadero. En este proceso los estudiantes se reconocieron como responsables de esas situaciones y avanzar en la necesidad de asumir compromisos para ayudar a resolver el problema.

Analizadas las finalidades identificadas en las prácticas (en cada caso), se procede a relacionarlas con las identificadas en lo que expresan las profesoras.

\section{Relación entre los propósitos que las profesoras piensan y expresan acerca de la enseñanza de las ciencias sociales y lo que hacen en sus prácticas educativas}

De acuerdo con los resultados descritos en el análisis anterior, en la profesora del caso 1 hay divergencia entre lo que piensa y dice, con lo que hace en la práctica cuando enseña ciencias sociales en la educación primaria. Sus expresiones sobre las 
finalidades de la enseñanza de las ciencias sociales se aproximan a una perspectiva crítica (BENEJAM, 2002). A pesar de que enfatiza en la importancia de que los estudiantes aprendan a resolver problemas de la vida real, al observar la práctica, lo que hace es inducir a que los estudiantes memoricen contenidos declarativos que se transmiten a través de diferentes medios.

La concepción de la enseñanza del conocimiento social que se evidencia en esta práctica, dista de la perspectiva integradora de las ciencias sociales planteada en los lineamientos curriculares, se relaciona con una de las perspectivas de la enseñanza de los social studies identificada por Goodman y Adler (1985): social studies as school knowledge [Estudios sociales como conocimiento escolar]. En esta perspectiva, el aprendizaje se define como la adquisición pasiva de información, con poco tiempo para cuestionar, desafiar o analizar críticamente el conocimiento escolar. Esto es lo que ocurre en la práctica con los contenidos sobre las regiones naturales de Colombia. La profesora espera que los alumnos memoricen información específica por medio de las actividades realizadas durante el desarrollo de la unidad. Se observa la preocupación de la profesora por abordar los contenidos disciplinares previstos en el plan de estudios sin que haya reflexión frente a la utilidad de este conocimiento para la comprensión de la realidad social, terminado en una enseñanza transmisiva de los estudios sociales como la denomina Ross (2020).

Por el contrario, en el caso 2 hay coherencia entre lo que la profesora piensa y dice con lo que hace en la práctica cuando enseña el conocimiento social en la educación primaria. Los resultados obtenidos en la entrevista permiten identificar que las finalidades de la enseñanza del conocimiento social se aproximan a un enfoque crítico (BENEJAM, 2002). La profesora resalta la importancia de que los estudiantes aporten a la solución de problemas de sus comunidades, y cree que la enseñanza de las ciencias sociales debe hacerse a partir de casos y problemas, así como desarrollar proyectos para integrar los saberes de diferentes disciplinas.

Su práctica es coherente con lo que expresa la profesora sobre cómo deben enseñarse las ciencias sociales, y se ubica también en un enfoque crítico al centrarse en un problema socioambiental abordado desde diferentes disciplinas, con el propósito de que los estudiantes lo comprendan, identifiquen múltiples causas, consecuencias, se comprometan con acciones para afrontar el problema y aportar a su solución.

Las concepciones de la profesora sobre la enseñanza del conocimiento social cuyas finalidades se ubican en un enfoque crítico, se mueven entre dos de las perspectivas de los social studies identificadas por Goodman y Adler (1985). El hecho de abordar la enseñanza del conocimiento social desde un problema socioambiental para integrar diferentes disciplinas, es muestra de 
una concepción de las ciencias sociales como the great connection [la gran conexión]. La profesora se preocupó por promover la indagación y la reflexión entre sus estudiantes, integró diferentes disciplinas y logró que se involucraran en el aprendizaje, aunque haya priorizado el conocimiento natural sobre el conocimiento social. Además, los llevó a comprometerse con acciones para generar cambios en sus comunidades relacionados con el problema abordado en las clases. Aquí se identifica una concepción de las ciencias sociales como acción social (social studies as social action), expuesta antes por los autores citados.

El caso 2 muestra que la coherencia entre el pensamiento y la acción, respecto a las finalidades de la enseñanza de las ciencias sociales desde el enfoque crítico, favorece el desarrollo de prácticas educativas innovadoras, consecuentes con las políticas curriculares que conciben unas ciencias sociales abiertas e integradoras, con la intención de superar la acumulación de información en historia y geografía para avanzar hacia la construcción de una ciudadanía crítica.

Los hallazgos de este trabajo plantean preocupaciones que deben considerarse en la formación de maestros, porque no bastan las buenas intenciones para desarrollar prácticas educativas innovadoras. Lo que ocurre en el caso 1 evidencia que sigue existiendo interés por el tratamiento de contenidos sustantivos: fechas, datos, conceptos y acontecimientos, privilegiados según las políticas curriculares en el siglo XIX e inicios del siglo XX, y se olvida la cercana relación que tienen las ciencias sociales con la construcción de identidades sociales, culturales, políticas, y con la transmisión de una memoria colectiva (CARRETERO, 2011; CARRETERO; ASENCIO; RODRÍGUEZMONEO, 2012).

En el caso 1, la enseñanza de las ciencias sociales se aleja de las ciencias abiertas e integradas, de las que se habla en los lineamientos curriculares (REPÚBLICA DE COLOMBIA, 2002). Hay un interés por abordar muchos contenidos sobre las regiones naturales en poco tiempo, lo que puede ser consecuencia del afán de la profesora por abordar el currículo prescrito desde parcelas disciplinares.

En el caso 2, se observa a una profesora que, en su primer año de ejercicio profesional, explora nuevas posibilidades de enseñar en torno a un problema que le permite integrar diferentes disciplinas, movilizar a los estudiantes hacia la comprensión de ese problema relacionado con lo que ven y viven en sus contextos. Se reconoce aquí una línea con enfoque abierto, flexible e integrado de las ciencias sociales, que propicia mayor acercamiento de la escuela a los problemas que afectan el entorno cercano y el planeta, y promueve mayor compromiso con el mundo en que viven los profesores y estudiantes (REPÚBLICA DE COLOMBIA, 2002). 


\section{Conclusiones}

Los resultados de los dos casos del estudio reflejan dos realidades que llevan a reflexionar, por un lado, sobre la formación inicial que ofrecen las universidades a quienes se desempeñarán como maestros en la educación básica primaria y, por otro lado, sobre la necesidad de indagar en el aula, de mirar el impacto de esas políticas gubernamentales que posiblemente estén desviando las discusiones de lo verdaderamente esencial: pensar los propósitos y las finalidades de la enseñanza de las ciencias sociales; definir el tipo de sociedad que queremos formar y qué acciones desarrollamos desde el ámbito educativo para lograrlo.

Es imprescindible que los profesores tengan claras las finalidades y los objetivos de lo que quieren enseñar para poder hacer una planificación educativa y, posteriormente, una práctica acorde con dichos propósitos. En la educación básica primaria, donde los profesores son generalistas, existe la ventaja de contar con una profesora que orienta todas las asignaturas del currículo, quien debe tener presente las finalidades de las ciencias sociales para tomar decisiones sobre los contenidos, los tiempos, espacios y demás aspectos relacionados con la práctica educativa, que aporten a la formación de ciudadanos críticos, siendo consecuente con los principios orientadores de los lineamientos curriculares.

En esencia, lo identificado en la reglamentación de la enseñanza de la historia y las ciencias sociales en Colombia y lo encontrado en los dos casos analizados, presenta contradicciones, tanto entre los documentos oficiales, como entre estos y lo que los profesores noveles generalistas piensan y llevan a la práctica, lo cual no siempre contribuye a los propósitos enunciados en la Ley general de educación y en los lineamientos curriculares, que propenden por la formación de ciudadanos críticos con el propósito de reconstruir la sociedad de maneras más equitativas y socialmente justas. 


\section{REFERENCIAS}

ACEVEDO, Álvaro; SAMACÀ, Alonso. La política educativa para la enseñanza de la historia de Colombia (1948-1990): de los planes de estudio por asignaturas a la integración de las ciencias sociales. Revista Colombiana de Educación, [s.l.], n. 62, p. 221-244, 2012.

ARIAS, Diego. La enseñanza de las ciencias sociales en Colombia: lugar de las disciplinas y disputa por la hegemonía de un saber. Revista de Estudios Sociales, Bogotá, n. 52, p. 134-146, 2015.

BENEJAM, Pilar. Las finalidades de la Educación Social. In: BENEJAM, Pilar; PAGÈS, Joan (coord.). Enseñar y aprender Ciencias Sociales, geografía e historia en la educación secundaria. Barcelona: Horsori, 2002. p. 33-52.

CARRETERO, Mario. Constructing Patriotism: Teaching history and memories in global world. Charlotte, NC: Information Age Publishing, 2011.

CARRETERO, Mario; ASENCIO, Mikel; RODRÍGUEZ-MONEO, María (eds.). History education and the construction of national identities. Charlotte, NC: Information Age Publishing, 2012.

DOGAN, Matei; PAHRE, Robert. Las nuevas ciencias sociales. La marginalidad creadora. Ciudad de México: Grijalbo, 1993.

DURKHEIM, Èmile. Las reglas del método sociológico. Buenos Aires: Orbis, 1986.

FLICK, Uwe. Introducción a la investigación cualitativa. 3. ed. Madrid: Morata, 2012.

FLICK, Uwe. La gestión de la calidad en Investigación Cualitativa. Madrid: Morata, 2014.

GIBBONS, Michael et al. La nueva producción del conocimiento. La dinámica de la ciencia y la investigación en las sociedades contemporáneas. Barcelona: Ediciones Pomares, 1997.

GOODMAN, Jesse; ADLER, Susan. Becoming an Elementary Social Studies Teacher: A Study of Perspectives. Theory and Research in Social Education, [s. l.], v. 13, n. 2, p. 1-20, 1985.

LEVSTIK, Linda; TYSON, Cynthia. Handbook of research in social studies education. New York: Routledge, 2008.

OROZCO, Juan. Estándares, enseñanza de las ciencias y control político del saber. Nodos y Nudos, [s. l.], v. 2, n. 17, p. 4-15, 2004. Disponible en: https://revistas.pedagogica.edu. co/index.php/NYN/article/view/1227. Acceso en: 10 enero 2020.

PATTON, Michael. Qualitative Evaluation and Research Methods. London: Sage, 1990.

PORLÁN, Rafael. Enseñanza Universitaria. Cómo mejorarla. Madrid: Morata, 2017. 
REPÚBLICA DE COLOMBIA. Constitución Política de Colombia de 1991. Gaceta Constitucional, Santafé de Bogotá, DC, n. 116, 20 jul. 1991. Disponible en: http://www. secretariasenado.gov.co/index.php/constitucion-politica. Acceso en: 28 nov. 2019.

REPÚBLICA DE COLOMBIA. Ministerio de Educación Nacional. Decreto 2388 de julio 15 de 1948, por el cual se intensifica la enseñanza de la historia patria y se dictan otras disposiciones. Diario oficial: Bogotá, n. 26779, 28 jul. 1948. Disponible en: https://www. mineducacion.gov.co/1759/articles-103421_archivo_pdf.pdf. Acceso en: 12 dic. 2019.

REPÚBLICA DE COLOMBIA. Ministerio de Educación Nacional. Decreto 1002 de abril 24 de 1984, por el cual se establece el Plan de Estudios Para la Educación Preescolar, Básica (Primaria y Secundaria) y Media Vocacional de la Educación Formal Colombiana. Diario oficial: Bogotá, n. 36615, 18 mayo 1984. Disponible en: https://www. mineducacion.gov.co/1621/articles-103663_archivo_pdf.pdf. Acceso en: 12 dic. 2019.

REPÚBLICA DE COLOMBIA. Ley 115 de 1994. Ley General de Educación. Diario Oficial: Bogotá, año CXXIX, n. 41214, p. 1, 8 feb. 1994. Disponible en: https://www. mineducacion.gov.co/1621/articles-85906_archivo_pdf.pdf. Acceso en: 13 dic. 2019.

REPÚBLICA DE COLOMBIA. Ministerio de Educación Nacional. Lineamientos curriculares de Ciencias sociales en la educación básica. Bogotá: MEN, 2002. Disponible en: https://www.mineducacion.gov.co/1759/w3-article-89869.html?_noredirect=1. Acceso en: 16 dic. 2019.

REPÚBLICA DE COLOMBIA. Ministerio de Educación Nacional. Estándares Básicos de Competencias en Lenguaje, Matemáticas, Ciencias y Ciudadanas. Guía sobre lo que los estudiantes deben saber y saber hacer con lo que aprenden. Bogotá: MEN, 2006. Disponible en: https://www.mineducacion.gov.co/1621/articles-340021_recurso_1.pdf. Acceso en: 16 dic. 2019.

REPÚBLICA DE COLOMBIA. Ministerio de Educación Nacional. Derechos básicos de aprendizaje. Bogotá: MEN, 2016. Disponible en: http://aprende.colombiaaprende.edu. co/sites/default/files/naspublic/DBA_C.Sociales.pdf. Acceso en: 16 dic. 2019.

REPÚBLICA DE COLOMBIA. Ley 1874 de diciembre 27 de 2017, por la cual se modifica parcialmente la Ley General de Educación, Ley 115 de 1994, y se dictan otras disposiciones. Diario Oficial: Bogotá, año CLIII, n. 50459, 27 dic. 2017. Disponible en: https://www.mineducacion.gov.co/1759/w3-article-381646.html?_noredirect=1. Acceso en: 19 dic. 2019.

RODRÍGUEZ, José; GARZÓN, Juan. Rutas pedagógicas en la enseñanza y el estudio de la historia. In: RODRÍGUEZ, José (ed.). Rutas pedagógicas de la enseñanza de la historia en la educación básica de Bogotá. Bogotá: Universidad Nacional de Colombia, 2004. p. 23-65.

ROSS, Wayne. History and social studies curriculum. In: OXFORD Research Encyclopedia of Education. Oxford: Oxford University Press, 2020. 
SÁNCHEZ VÁSQUEZ, Nubia Astrid. Debates y discursos en torno a las ciencias sociales escolares entre 1984 y 2010 en Colombia. Uni-pluri/versidad, Medellín, v. 13, n. 2, p. 69-80, 2013.

SANTISTEBAN, Antoni; PAGÈS, Joan (coord.). Didáctica del conocimiento del medio social y cultural en la educación primaria. Madrid: Síntesis, 2011.

SHULMAN, Lee. Conocimiento y enseñanza: Fundamentos de la nueva reforma. Revista de Currículum y formación del profesorado, San Francisco, CA, v. 9, n. 2, p. 1-22, 2005.

STRAUSS, Anselm; CORBIN, Juliet. Bases de la investigación cualitativa. Medellín: Universidad de Antioquia, 2012.

WALLERSTEIN, Immanuel (coord.). Abrir las ciencias sociales. Ciudad de México: Siglo XXI, 1999.

YIN, Robert. Case Study Research: Design and Methods. 4. ed. London: Sage, 2009.

Texto recibido el $04 / 10 / 2020$.

Texto aprobado el 25/02/2021. 